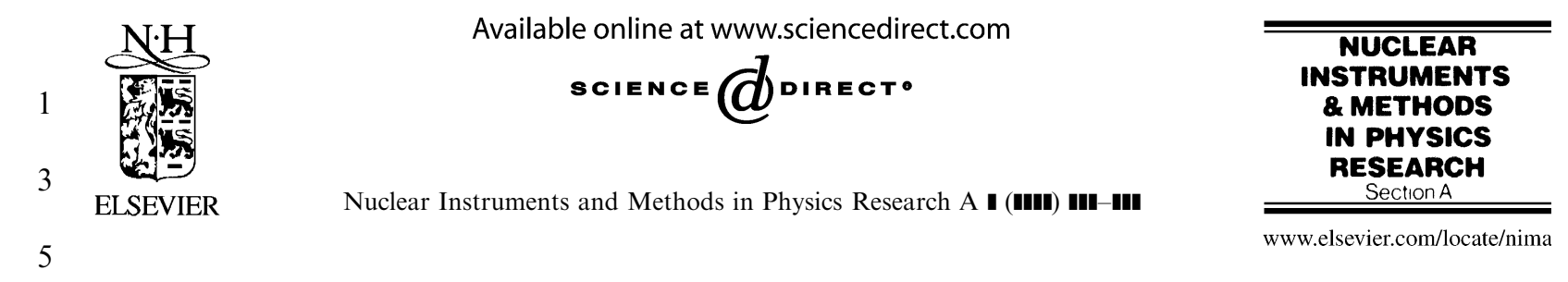

\title{
The effect of protons on E2V technologies L3Vision CCDs
}

\author{
D.R. Smith ${ }^{\mathrm{a} * *}$, A.D. Holland ${ }^{\mathrm{a}}$, M.S. Robbins ${ }^{\mathrm{b}}$ \\ ${ }^{a}$ Space Research Centre, University of Leicester, University Road, Leicester, LE1 7RH, UK \\ ${ }^{\mathrm{b}}$ E2V Technologies, Waterhouse Lane, Chelmsford, CM1 2QU, UK
}

\section{Abstract}

The effect of different $10 \mathrm{MeV}$ equivalent proton fluences on the performance of E2V Technologies (formerly Marconi applied technologies, formerly EEV) L3Vision Charge Coupled Devices (CCDs) was investigated. The first experimental radiation damage results of the L3Vision device are presented, with emphasis given to the analysis of damage to the gain register of the device. Changes in dark current and generation of bright pixels in the CCD image, store, readout register and gain register as a result of proton irradiation are reported and viewed in light of the potential use of the device in space-based applications. (C) 2003 Published by Elsevier B.V.

Keywords: Charge coupled device; Proton; Damage; Radiation; Low light level; L3Vision

\section{Introduction}

The E2V technologies low light level (L3Vision) Charge Coupled Devices (CCD), uses a novel method of charge readout that is capable of an equivalent output noise of less than one electron at pixel rates of over $11 \mathrm{MHz}[1,2]$. The CCD is an inverted mode operation, frame transfer device, that has a standard readout register followed by a 'gain' register that multiplies the signal charge before it is converted to a voltage. The image and store sections of the CCD are each $591 \times 296$ pixels, while the readout and gain registers are each 591 pixels in length plus a few reference pixels. Fig. 1 shows the geometrical layout of the L3Vision device. The pixels in the image, store and readout register of the device measure $20 \mu \mathrm{m} \times$

*Corresponding author. Tel.: +44-116-252-3519; fax: +44116-252-2464.

E-mail address: drs@star.le.ac.uk (D.R. Smith).
$30 \mu \mathrm{m}$, while pixels in the gain register measure $40 \mu \mathrm{m} \times 30 \mu \mathrm{m}$ to handle the potentially larger signal charge after gain.

The L3Vision device is suited to applications where light levels are very low and therefore has potential for use in space-based applications when looking at faint sources. However, the avalanche regions could be susceptible to catastrophic breakdown failure as a result of radiation damage. To assess this potential, two L3Vision devices were irradiated with proton fluences representative of mission fluences expected to be received by typical orbiting spacecraft [3].

\section{Experimental method}

Irradiation of each L3Vision CCD was carried out using the accelerator facility at Birmingham University, UK. Prior to irradiation of the CCDs, proton beam uniformity over the target region was 
examined using a photodiode in pulse counting mode, with the result displayed on a spectrum analyser. Across the CCD area, the beam uniformity was found to be $\pm 15 \%$. The flux reaching the photodiode in $1 \mathrm{~min}$ was measured several times to calibrate the beam flux, to measure the stability of the beam and to ensure the required proton doses could be given over a suitable time scale. The error associated with the dosimetry was estimated to be $\sim 20 \%$.

Once the proton beam characteristics were determined, the two CCDs were irradiated one after the other at a temperature of $22^{\circ} \mathrm{C}$. In each case the target $\mathrm{CCD}$ was mounted in a vacuum chamber attached to the end of the beamline, with all pins grounded to avoid potential static damage.

Aluminium shields were used to cover parts of the CCDs that were to be kept unirradiated as control areas. Fig. 2 shows the area of each device irradiated and the $10 \mathrm{MeV}$ equivalent proton dose each area received. The mean energy of the proton beam was $6.5 \mathrm{MeV}$.

For each irradiation the photodiode was positioned $\sim 2 \mathrm{~cm}$ in front of the shielded section of the CCD to accurately monitor the proton flux reaching the CCD in real time.

The whole of the readout and gain registers, and half of the image and store sections, of device 00463-10-12 were irradiated with a $10 \mathrm{MeV}$ equivalent proton fluence of $5.1 \times$ $10^{8}$ protons $\mathrm{cm}^{-2}$. A $10 \mathrm{MeV}$ equivalent proton fluence of $2.0 \times 10^{9}$ protons $\mathrm{cm}^{-2}$ was given to the

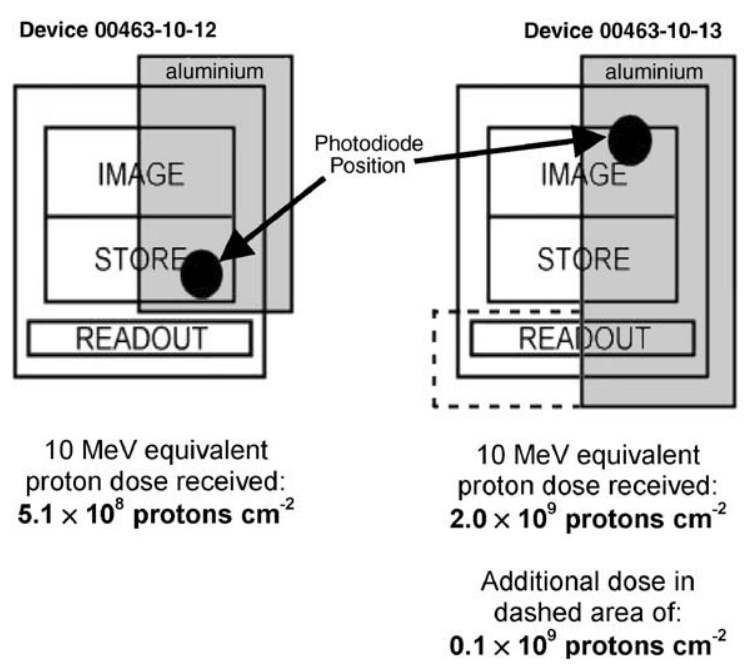

Fig. 2. This diagram shows the irradiated areas of the two CCDs and gives the dose received by each area.

left half of device $00463-10-13$, with an additional dose of $0.1 \times 10^{9}$ protons $\mathrm{cm}^{-2}$ given to just the left half of the readout and gain registers.

\section{Experimental results}

After irradiation, both CCDs were functional and, at a temperature of $22^{\circ} \mathrm{C}$, showed increased dark current and bright pixel counts comparable to those observed in CCDs subjected to similar proton doses $[4,5]$.

For CCD 00463-10-13, a sequencer program was used to readout only the readout and gain register pixels of the device. The image and store sections of the CCD were back-clocked to avoid thermal leakage current from the image and store sections entering into the readout register. A series of short $3 \mathrm{~ms}$ row integrations were then taken. Fig. 3 shows an accumulation of 200 such rows, together with annotations indicating the different device and proton exposure regions.

Fig. 4 gives the sum average of the rows in the recorded image. The figure has four sections, which are from right to left: non-irradiated readout register, irradiated readout register, irradiated gain register, and non-irradiated gain register. The slope of the signal in the gain register and the 


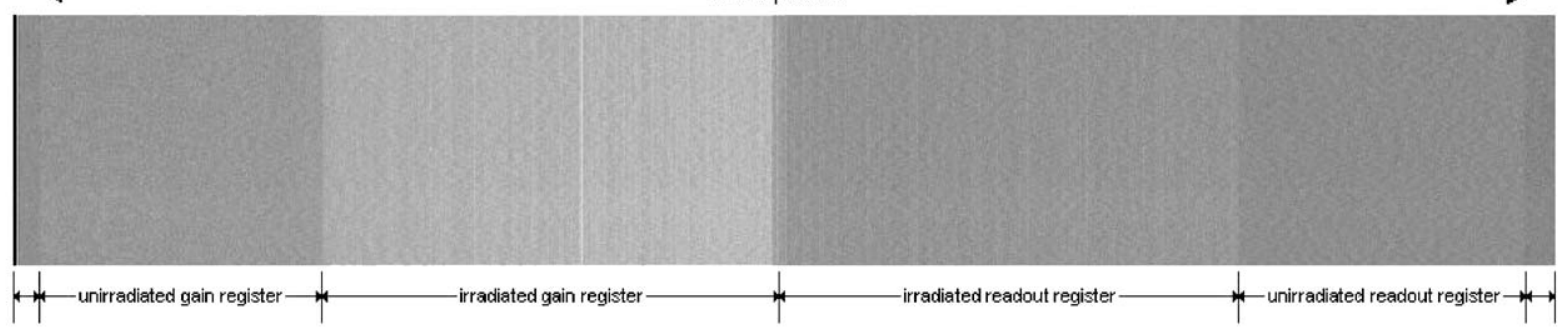

Fig. 3. An image taken using a sequencer program that only reads out pixels in the readout and gain registers of the device. The irradiated and unirradiated sections of the readout register can be seen, along with under and overscan pixels.

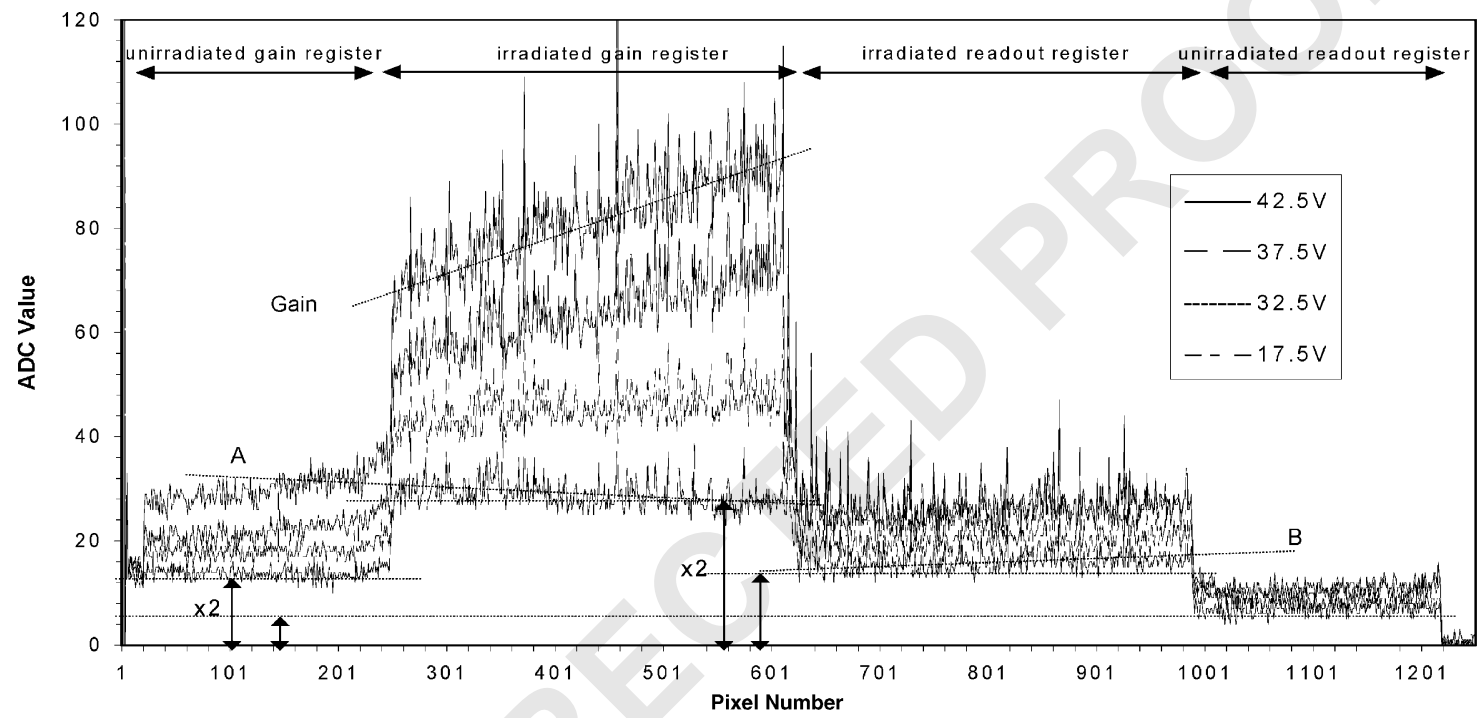

Fig. 4. The effect of different applied voltage on the L3Vision readout/gain register. Slopes $A$ and $B$ show an indication of proton beam non-uniformity with low applied voltage. The gain is seen to increase sharply once the applied voltage is increased above $30 \mathrm{~V}$.

increase in number of bright pixels and base dark current level due to proton irradiation can all be seen in this figure. The factor $\sim 2$ increase in dark current level between the gain register and readout register is due to the factor $\sim 2$ increase in pixel size from those in the readout register compared to those in the gain register. At low applied voltage levels an indication of proton beam non-uniformity, slopes $A$ and $B$ in Fig. 4, can be seen.

Comparison of the measured post irradiation gain curves with those on the L3Vision CCD data sheet show that the irradiations have not significantly affected the behaviour of the device (Fig. 5).

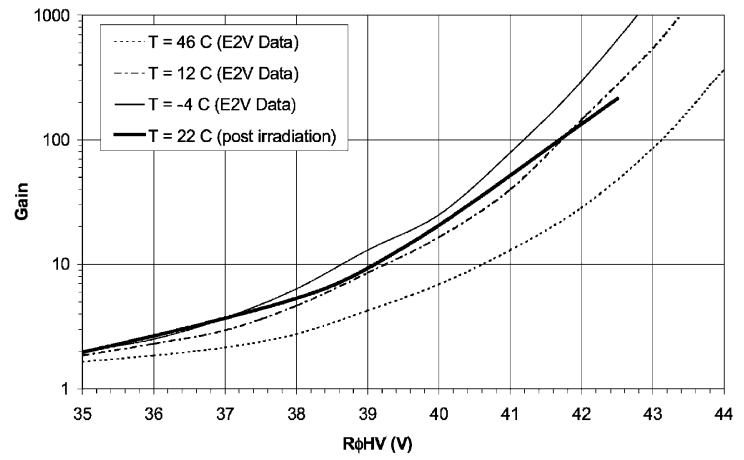

Fig. 5. Measured variation of gain with applied voltage post irradiation. Gain curves from the E2V Technologies L3Vision CCD data sheet are shown for comparison. 


\section{4. Conclusions}

3 After irradiation with protons, the L3Vision device is found to operate normally, with the

5 resulting change in dark current and number of bright pixels comparable to previous proton

7 irradiation studies. The behaviour of the gain register did not alter as a result of proton

9 irradiation. Bright pixels generated in the gain register were found to increase in amplitude in the

11 same way as the normal gain register pixels, showing no evidence of field enhancement effects.

13 It is therefore assumed the observed bright pixels are not located in the vacinity of a high field

15 avalanche region.

After studying the effects of proton irradiation

17 on two L3Vision devices, there appear to be no problems that would inhibit the use of the device

19 for space-based applications. There is however a need to irradiate further devices to obtain better

21 statistics to deduce if emission sites generated in the high-field regions of the gain register pixels can

23 cause device failure. This study cannot be carried out by irradiating a single device to a high fluence

25 as this would result in all the pixels in the gain register becoming bright.

27 Previous proton irradiation studies on conventional devices have shown that irradiation with the

29 device unbiased, as in this study, induces signifi- cantly lower voltage shifts than if the device were operational during the irradiations. Further ionising irradiations are required to establish the magnitude of these voltage shifts and their effect on L3Vision device performance.

\section{Acknowledgements}

The authors would like to thank Mike Smith at Birmingham University, UK, for his assistance during the experimental phase of this study, and E2V Technologies for the CCDs used in this work.

\section{References}

[1] P. Jerram, P. Pool, R. Bell, D. Burt, S. Bowring, S. Spencer, M. Hazelwood, I. Moody, N. Catlett, P. Heyes, Sensors and camera systems for scientific, industrial, and digital photography applications II, Proc. SPIE 4306 (2001).

[2] C.D. Mackay, R.N. Tubbs, R. Bell, D. Burt, I. Moody, Proc. SPIE 4306 (2001) 289.

[3] A. Holmes-Siedle, S. Watts, A. Holland, Final Report on ESTEC Contract No. 8815/90/NL/LC(SC), Brunel University, UK, 1995.

[4] D.R. Smith, A.D. Holland, M.S. Robbins, R.M. Ambrosi, I.B. Hutchinson, Proc. SPIE 4851 (2002).

[5] R.M. Ambrosi, A.D.T. Short, A.F. Abbey, A.A. Wells, D.R. Smith, Nucl. Instr. and Meth. A 482 (2002) 644. 